duration of ECMO or MV between patients with and without positive virology.

Conclusion In a single regional intensive care unit we demonstrate that requirement for ECMO due to acute asthma is associated with female gender, younger age and positive virology on admission. To our knowledge, this is the first case series analysing factors relating to ECMO use in asthma in the United Kingdom. It highlights the role of respiratory viruses in near-fatal exacerbations and the need for novel anti-viral approaches to reduce morbidity and mortality. Further research is needed in this population to identify whether differences in underlying inflammatory mechanisms exist that may explain the development of such severe events.

\section{P133 SAFETY AND EFFECTIVENESS OF INFLUENZA VACCINES IN PEOPLE WITH ASTHMA: A SYSTEMATIC REVIEW AND META-ANALYSIS}

${ }^{1}$ E Vasileiou, ${ }^{1} \mathrm{~A}$ Sheikh, ${ }^{2,3} \mathrm{C}$ Butler, ${ }^{1} \mathrm{~K}$ El Ferkh, ${ }^{1} \mathrm{CR}$ Simpson. ${ }^{1}$ Asthma UK Centre for Applied Research, Usher Institute of Population Health Sciences and Informatics, The University of Edinburgh, Edinburgh, UK; ${ }^{2}$ Nuffield Department of Primary Care Health Sciences, Oxford University, Oxford, UK; ${ }^{3}$ Institute of Primary Care and Public Health, Cardiff University, Cardiff, UK

\subsection{6/thoraxjnl-2016-209333.276}

Introduction and objectives Influenza vaccination is offered annually in the UK to high-risk individuals such as those with asthma as a preventive measure against influenza infection and influenza-related complications. However, the effectiveness and safety of influenza vaccination in people with asthma is not well established. ${ }^{1}$

Methods We conducted a systematic review and meta-analysis assessing the overall quality of evidence using the GRADE methodology. Published literature was searched through 13 electronic databases from Jan 1970 to Jan 2016 for clinical trials and epidemiological studies. Unpublished or ongoing literature was searched through references and citations of key publications, and by contacting influenza vaccine manufacturers. The screening for eligible studies, data extraction and quality appraisal was conducted by two reviewers independently. Separate meta-analyses were undertaken for observational and experimental evidence using random-effects models.

Results We identified 35 eligible studies, and four contributed to the meta-analyses. Risk of bias was high for one randomised controlled trial (RCT), unclear for 11 RCTs, and low for eight RCTs. The quality of five non-RCTs, four cohorts, and two case-control studies was strong. Moderate quality was found for one nonRCT, and three cohort studies. In people with asthma, pooled vaccine effectiveness (VE) was $45 \%$ (OR: 0.55 ; 95\% CI: 0.44 to 0.69 ; I $2=0 \%$ ) for laboratory confirmed influenza. Pooled effectiveness of live vaccines was $81 \%$ (RR: 0.19 ; 95\% CI: 0.06 to $0.67 ; \mathrm{I} 2=0 \%$ ) for influenza infection (confirmed by cell culture or rise in antibody titre) and $72 \%$ (RR: $0.28 ; 95 \%$ : 0.10 to 0.80 ; $\mathrm{I} 2=0 \%$ ) for influenza-like illness. VE was also observed against asthma attacks. No increased risk of vaccine-induced asthma symptoms and attacks was identified. The quality of the body of evidence was considered very low for all outcomes.

Conclusions Evidence on VE in people with asthma against influenza, asthma exacerbations, and other clinical outcomes is limited and of very low quality. Thus, better quality evidence is required, especially in adults with asthma. Vaccination with inactivated or live vaccines was found to be safe and well tolerated in patients with asthma.

\section{REFERENCE}

1 Cates CJ, Rowe BH. Vaccines for preventing influenza in people with asthma. Cochrane Database Sys Rev 2013;2:Cd000364

\section{P134 METHACHOLINE CHALLENGE TO DEMONSTRATE THERAPEUTIC EQUIVALENCE OF TERBUTALINE VIA DIFFERENT TURBUHALER DEVICES IN PATIENTS WITH MILD TO MODERATE ASTHMA: APPRAISAL OF A PHASE III, FOUR-WAY CROSSOVER DESIGN}

${ }^{1} \mathrm{~L}$ Bjermer, ${ }^{2} \mathrm{G}$ Gauvreau, ${ }^{3} \mathrm{D}$ Postma, ${ }^{2} \mathrm{P}$ O'Byrne, ${ }^{3} \mathrm{M}$ van den Berge, ${ }^{4} \mathrm{~L}-\mathrm{P}$ Boulet, ${ }^{5} \mathrm{O}$ Beckman, ${ }^{5} \mathrm{~T}$ Persson, ${ }^{5} \mathrm{~J}$ Roman, ${ }^{5} \mathrm{M}$ Carlholm, ${ }^{5} \mathrm{~K}-\mathrm{M}$ Schutzer, ${ }^{5} \mathrm{G}$ Eckerwall. ${ }^{1}$ Skane University Hospital, Lund, Sweden; ${ }^{2}$ McMaster University, Hamilton, Canada; ${ }^{3}$ University of Groningen, Groningen, The Netherlands; ${ }^{4}$ Quebec Heart and Lung Institute, Quebec, Canada; ${ }^{5}$ AstraZeneca RandD, Gothenburg, Sweden

\subsection{6/thoraxjnl-2016-209333.277}

Background/objective To demonstrate therapeutic equivalence of terbutaline via two different Turbuhaler ${ }^{\circledR}$ devices by evaluating its protective effect against methacholine-induced bronchoconstriction in patients with stable asthma.

Methods In this double-blind, double-dummy, multicentre, single-dose, 4-way crossover study, patients with stable, mild-tomoderate asthma (FEV1 $\geq 80 \%$ predicted normal) were randomised to 0.5 or $1.5 \mathrm{mg}$ terbutaline via either Turbuhaler ${ }^{\circledR} \mathrm{M} 3$ or M2 followed by a methacholine challenge test. Primary outcome variable: concentration of methacholine causing a $20 \%$ drop in FEV1 (PC20). Patients had to have a PC20 methacholine $<8$ $\mathrm{mg} / \mathrm{mL}$, reproducible after 2 weeks, and a stable baseline FEV1 at all visits (90-110\% of enrolment value).

Results 60 patients were randomised to treatment and completed the study. There was a clear dose-response for both devices. The within-device ratios (1.5 mg: $0.5 \mathrm{mg}$ ) were 1.79 and 1.87 for Turbuhaler M3 and M2, respectively (both p < 0.001). The between-devices ratio (M3:M2) was 0.92 (95\% CI: 0.75-1.13) for $0.5 \mathrm{mg}$ and $0.88(95 \%$ CI $0.72-1.08)$ for $1.5 \mathrm{mg}$. Both CIs lie inside the interval $(0.67-1.50)$, which was the pre-specified condition for equivalent effect.

Conclusions Bronchoprotection with PC20 as the outcome measure in a standardised methacholine challenge model proved to be a useful design to show therapeutic equivalence between devices in patients with mild to moderate asthma. This model provides robust reproducible data, involves smaller patient numbers with fewer dropouts resulting in reduced costs versus a conventional efficacy study.

\section{Disease Progression and Burden in Obstructive Lung Disease}

\section{P135 TREATMENT OF LUNG DISEASE IN ALPHA-1 ANTITRYPSIN DEFICIENCY: A SYSTEMATIC REVIEW}

${ }^{1}$ RG Edgar, ${ }^{2} \mathrm{M}$ Patel, ${ }^{3} \mathrm{~S}$ Bayliss, ${ }^{4} \mathrm{E}$ Sapey, ${ }^{4}$ AM Turner. 'University Hospitals Birmingham NHS Foundation Trust, Birmingham, UK; ${ }^{2}$ Heart of England NHS Foundation Trust, Birmingham, UK; ${ }^{3}$ Institute of Applied Health Research, University of Birmingham, Birmingham, UK; ${ }^{4}$ Institute of Inflammation and Ageing, University of Birmingham, Birmingham, UK

\subsection{6/thoraxjnl-2016-209333.278}

Introduction Alpha-1 Antitrypsin Deficiency (AATD) is a rare genetic condition predisposing individuals to COPD. The 


\section{Withdrawn notices}

Bjermer L, Gauvreau G, Postma D, et al. P134 Methacholine challenge to demonstrate therapeutic equivalence of terbutaline via different turbuhaler devices in patients with mild to moderate asthma: appraisal of a phase iii, four-way crossover design. Thorax 2016;71:A155. doi:10.1136/thoraxjnl-2016-209333.277

This abstract has been withdrawn.

Thorax 2017;72:287. doi:10.1136/thoraxjnl-2016-209333.277wthn 